\title{
When a Nova Becomes Old
}

\author{
A. Ederoclite ${ }^{1}$, C. Tappert ${ }^{2}$, L. Schmidtobreick ${ }^{3}$, N. Vogt $^{2}$ \\ ${ }^{1}$ Centro de Estudios de Física del Cósmos de Aragón (CEFCA), Plaza San Juan 1, Planta 2, 44001, Teruel, Spain \\ ${ }^{2}$ Instituto de Fúsica y Astronomía, Universidad de Valparaiso, Av. Gran Bretaña 1111, Valparaiso, Chile \\ ${ }^{3}$ European Southern Observatory, Alonso de Cordova, Santiago, Chile
}

Corresponding author: aederocl@cefca.es

\begin{abstract}
Here we present the preliminary results of a project aimed at unveiling the nature of classical novae decades after their eruption. The ultimate goal of this project is to describe the population of cataclysmic variables which give rise to nova explosions. So far, in four years of observations, we have concentrated on novae in the Southern hemisphere, where we increased by $100 \%$ the amount of objects spectroscopically confirmed and increased by $1 / 5$ the amount of objects with known orbital period.
\end{abstract}

Keywords: cataclysmic variables - classical novae - optical - spectroscopy - photometry.

\section{Introduction}

Classical Novae (CNe) are the result of a thermonuclear runaway on the surface of a white dwarf which is accreting mass from a less evolved companion (for a review, see Bode \& Evans, 2008). It is common to define CNe as a "subclass of cataclysmic variables" which is a fair statement in both historical and observational terms but it is not quite physically appropriate: a $\mathrm{CN}$ explosion is a phase during the life time of a cataclysmic variable $(\mathrm{CV})$.

According to the "hibernation scenario" (Shara et al., 1986), post-nova CVs are expected to go through a phase of high mass transfer (triggered by the irradiation of the hot WD) and, eventually, the system gets to separate and spends $90-99 \%$ of the inter-explosion time in a quiescent "detached" state (Prialnik 1986). It is clear that the use of the term "quiescence" for an oldnova system is very vague and it is the reason why it is used in quotes throughout this work. For a review on old novae, see Pagnotta (2013).

This project started four years ago trying to answer some fundamental questions:

- how does the population of old novae look like?

- how does the population of old novae compare to CVs?

- do short-period old novae exist in a significant number?

- what is the role of magnetic fields on the CNoutburst?
Despite their brightness at maximum light, the study of novae in "quiescence" is still largely incomplete (again, see Pagnotta, 2013). It is important to note that, before this project started, only 39 old novae (out of the 204 novae which exploded before 1980) had a measured orbital period. So far, our project has focused mostly on the Southern Hemisphere (i.e. targets with $\delta<20^{\circ}$ ), where, before the start of our project, out of 153 reported novae, 34 lacked identification of the target in "quiescence", 9 were confirmed but their period was unknown and 24 had a known orbital period. The remaining 86 did not even have a candidate.

Here we describe the methodology and some early results related with the population which has been recovered so far. For the description of selected targets, see Schmidtobreick \& Tappert (2013) and Tappert et al. (2013c) .

\section{The Methodology}

Our project consists of three phases: the photometric identification of the nova candidate, the spectroscopic confirmation and the period determination through time-series spectroscopy.

The target list has been derived mainly from the Downes et al. (2005) catalogue. We selected those novae which had exploded before 1980. The selection is made to allow some time after the nova explosion for the characteristics of the underlying $\mathrm{CV}$ to become accessible. 

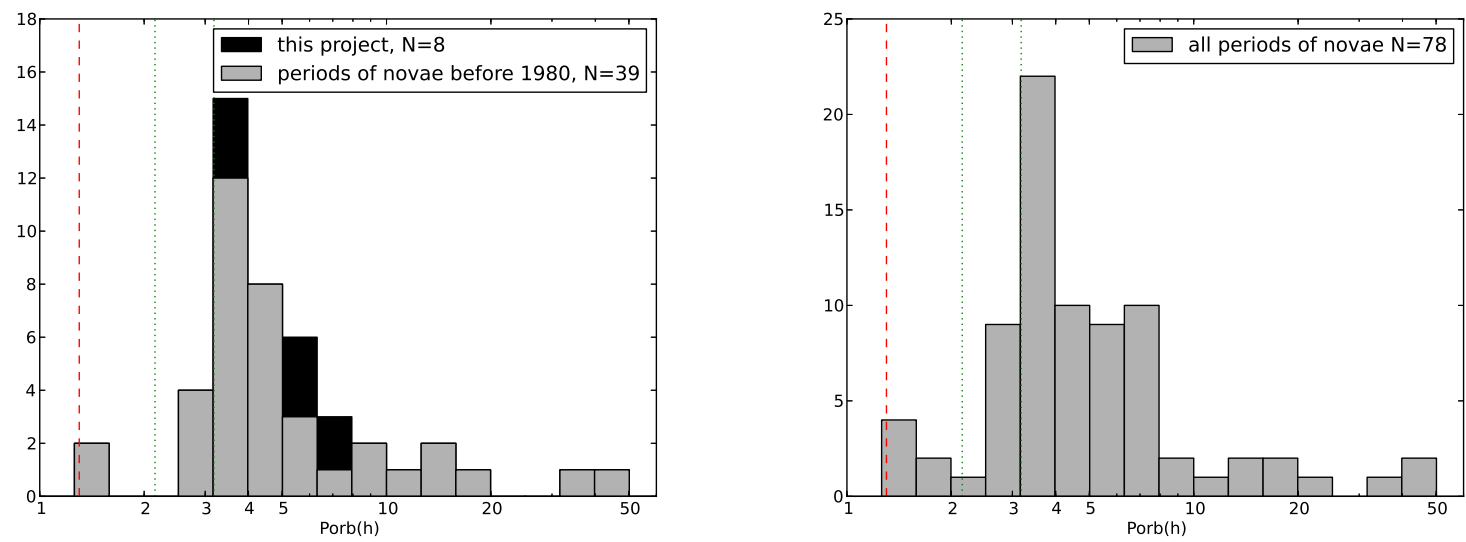

Figure 1: Left: Period histogram of novae which exploded before 1980. The black histogram is the addition from this project. Right: Period histogram of all novae (i.e. including those which exploded after 1980). The dotted vertical lines refer to the period gap and the dashed vertical line to the period minimum.

We do not include in our sample recurrent novae (RNe). These represent thermonuclear explosions analogous to $\mathrm{CNe}$ but which occur on much shorter recurrence times. The large majority of those rare stars (only a dozen is known in our Galaxy) harbour well-evolved secondary stars which sets them somewhat apart from other CVs. As our project primarily originated with the idea of testing the predictions of the hibernation scenario, $\mathrm{RNe}$ are not included in our analysis.

We obtain $U B V R$ photometry of the region of sky where a nova has been reported. Here we are taking advantage of the fact that an old nova, as any CV, has a spectrum which is the sum of three components (the white dwarf, the main sequence companion and the accretion disk). This is the same discovery strategy which is being used in large photometric surveys, like SDSS (see Szkody, 2013).

We obtain spectroscopy of those targets whose location in the colour-colour diagram, does not follow the one of the stars. This is "weighted" by the distance from the reported position (i.e. not necessarily the bluest object in the field is the first candidate for follow up spectroscopy, in case there is another object which is closer to the reported position). Low-resolution spectroscopy $\left(R=\frac{\lambda}{\Delta \lambda} \simeq 500\right)$ is enough to resolve CVs emission lines and to cover a spectral range $(\sim 4000-9000 \AA)$ which includes the most relevant lines for CV classification.

Time-series is obtained once the target has been spectroscopically confirmed. In some cases, time-series photometry is carried out (e.g. Tappert et al., 2013b) but, in general, we perform time-series spectroscopy with $R=\frac{\lambda}{\Delta \lambda} \simeq 2500$ in the $\mathrm{H} \alpha$ region.

In order to keep a log of the observations and share the results within a group which is geographically spread over two continents, an internal webpage is being maintained. Here we keep a list of our targets, observing logs, and description of the results for each target.

\section{$3 \quad$ Results}

\subsection{The period distribution}

The left panel of Fig.1 shows the histogram of the pre1980 novae. In grey, the periods that can be obtained in the literature and, in black, the addition due to this project. The right panel of Fig.1 shows the histogram of all the novae (also the ones which exploded after 1980). The periods of post-1980 novae are from Ritter \& Kolb (2003).

Qualitatively, the two histograms are very similar: there are few sources with periods within the period gap and a handful of sources below the period gap. There is a "clustering" of sources right above the period gap (just in the period region where SW Sex stars belong, see Schmidtobreick \& Tappert, 2013) and less sources at longer periods. This is an interesting result by itself, since our sample is selected on the basis that the targets are back in "quiescence". The only significant difference is the number of objects found in the 5 10 hours period range, which can be easily ascribed to small-number statistics.

As we already mentioned, very few objects are within (or below) the period gap. This is consistent with old novae being in a high-mass transfer phase, as predicted by the "hibernation" scenario. It is also worth noting that the overall shape is consistent with the modelling by Townsley \& Bildsten (2005), which assumes that novae occur mostly in high mass accretion rate systems. 


\subsection{The role of magnetic fields}

As we mentioned in the previous section, there are very few objects with periods $<3$ hours, and, as it can be seen from Fig.2, these objects are mostly magnetic. Among the 47 pre-1980 old novae with a measured period, only V1500 Cyg (1975) and CP Pup (1942) are catalogued as AM Her stars and have periods of 3.36 hours and 1.68 hours, respectively.

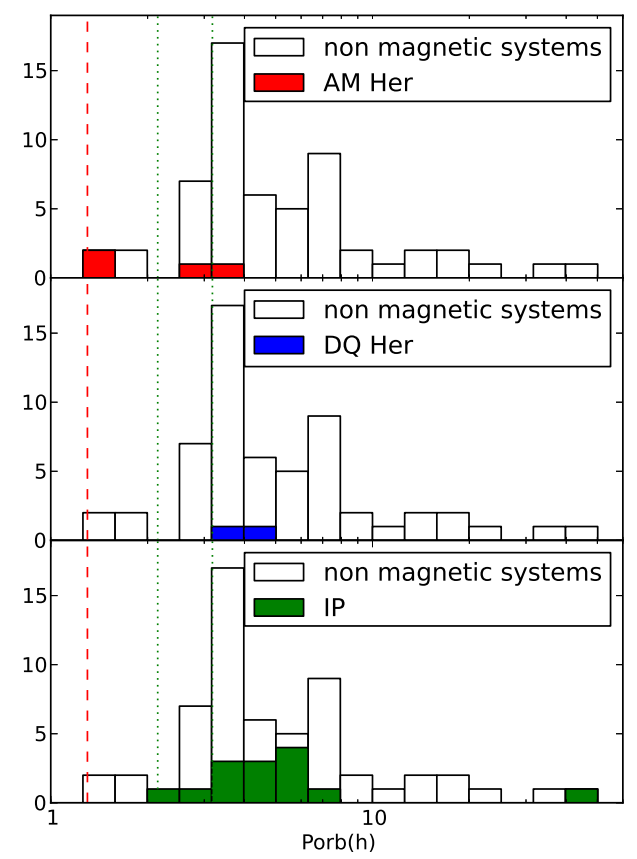

Figure 2: The histograms of the magnetic systems which are observed to have harboured a nova explosion. In white, for comparison, the histogram of nonmagnetic systems. In the upper panel, the AM Her, in the middle panel the DQ Her systems and, in the lower panel the intermediate polars. The classification is either from Ritter \& Kolb (2003) or from Tappert et al. (2012,2013a).

Although the number of magnetic systems is still small (hence we cannot draw firm conclusions) it is promising that polars and intermediate polars are located in a similar region of the period histogram as in the "classical" CV period histogram. Moreover, as mentioned by Tappert et al. (2013b), 7 out of 10 novae in the gap may be magnetic. This is in agreement with the population model by Townsley \& Bildstein (2005), who suggest that novae within the period gap are mostly magnetic. Araujo-Betancor et al. (2005) derive that the total ratio of known magnetic to nonmagnetic CVs is $\sim 22 \%$ (at all periods). The high number of magnetic
CVs within the gap, if confirmed, may imply a different evolution through the gap between magnetic and nonmagnetic systems.

\subsection{The explosion amplitude}

From Downes et al. (2005), one can derive that the average explosion amplitude is $12.57 \pm 2.47 \mathrm{mag}$. Combining our data with data from Downes et al. (2005), Ritter \& Kolb (2003), Duerbeck (1987), one can try to compare the explosion amplitude (i.e. the difference between the magnitude at maximum and the magnitude in "quiescence") with the rate of decline (see Fig.3). This has already been done by Vogt (1990). In Fig.3, we show the data from Vogt (1990) plus data from our project and post-1980 novae from Ritter \& Kolb (2003). It is interesting to note that our data points (the red squares) fall in the same region as the others. The straight line is the fit from Vogt (1990) to the points in his paper and is still valid with the inclusion of the new points.

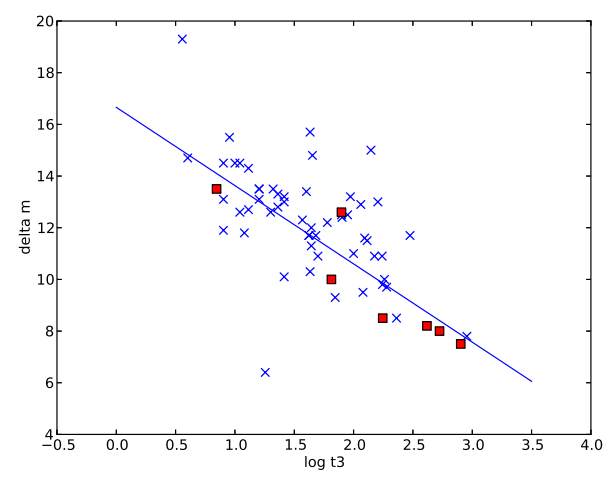

Figure 3: Rate of decline (as measured by the $t_{3}$ parameter) vs. explosion amplitude (difference between the magnitude at minimum and the magnitude at maximum). The red squares are from this project. The line is not a fit to the present data-set but to the data presented in Vogt (1990).

The two objects which seem to deviate from the relation are V1500 Cyg (1975) and V458 Vul (2007). The first one, as mentioned in the previous section, is an AM Her star. The second one showed a very irregular light curve, with three repeated peaks. These two examples suggest that the scatter in this relation is likely due to two independent causes: on one hand, the magnitude at "quiescence" depends on the structure of the accretion disk (if any) and the magnitude at maximum light (and the rate of decline) may be misestimated due to a series of factors (e.g. bad sampling or dust formation) which are quite typical in novae. Warner (1987) has already 
suggested that, for a better comparison of the explosion amplitude, one should take into account, at least, the orbit inclination of the system. Another important caveat is that some old novae are observed to decrease their "quiescent" brightness (see e.g. Vogt 1990, and references therein, or Johnson et al. 2013) and, therefore, Fig. 3 should be considered in a more dynamical context.

\section{Summary and Future Perspectives}

We have presented a project aimed at the recovery, classification and characterisation of classical nova systems decades after the explosion. During this project, which started four years ago, we have already significantly increased the number of identified and spectroscopically confirmed classical novae (novae without an identified progenitor decreased by $24 \%$ and the number of spectroscopically confirmed increased by $100 \%$ ). Finally, we have also increased by $1 / 5$ the number of pre-1980 oldnovae with known orbital period.

We plan to finalise the southern part of our project during the next two years and to focus on targets in the northern hemisphere. Once the program will be completed, this sample will be crucial in the study of the evolution of classical novae towards quiescence, the study of CN-progenitors as a subclass of the $\mathrm{CN}$ population and the development of of the "nova populations" framework (Della Valle 2002 and references therein).

For better sharing the results of our project, we are planning on providing access through the Virtual Observatory to the reduced data, thus sharing a database with basic parameters (position, brightness, type,...), the spectra used for characterisation and finding charts. This data release will represent a significant legacy for the $\mathrm{CV}$ community.

\section{Acknowledgement}

The CEFCA is funded by the Fondo de Inversiones de Teruel, supported by both the Government of Spain (50\%) and the regional Government of Aragón (50\%). This work has been partially funded by the spanish Ministerio de Ciencia e Innovación through the PNAYA, under grants AYA2006-14056 and through the
ICTS 2009-14. CT and NV acknowledge financial support by FONDECYT Regular grant 1120338.

\section{References}

[1] Araujo-Betancor, S. et al.: 2005, ApJ, 622, 589

[2] Bode, M. \& Evans, N.: 2008, "Classical Novae", 2nd Edition, Cambridge Astrophysics Series, No 43, Cambridge: Cambridge University Press, 2008

[3] Della Valle: 2002, AIPC, 637, 443

[4] Downes, R. et al.: 2005, JAD, 11, 2

[5] Duerbeck, H.: 1987, Space Sci. Rev. 45, 1 doi:10.1007/BF00187826

[6] Johnson, C. et al.: 2013, ApJ, 780L, 25

[7] Pagnotta, A.: 2013, this workshop

[8] Prialnik, : 1986, ApJ, 310, 222

[9] Ritter H., Kolb U. 2003, A\&A, 404, 301 doi:10.1086/164762 doi:10.1111/j.1365-2966.2012.21054.x

[10] Schmidtobreick, L. \& Tappert, C.: this workshop doi:10.1093/mnras/stt139

[11] Shara, M. et al. 1986 ApJ, 311, 163 doi:10.1093/mnras/stt 1747

[12] Szkody, P.: 2013, this workshop

[13] Tappert, C. et al.: 2012, MNRAS, 423, 2476 doi:10.1086/430594

[14] Tappert, C. et al.: 2013a, MNRAS, 431, 92 doi:10.1086/168866

[15] Tappert, C. et al.: 2013b, MNRAS, 436, 2412

[16] Tappert, C. et al.: 2013c, this workshop

[17] Townsley, D. \& Bildsten, L.: 2005, ApJ, 628, 395

[18] Vogt, N.: 1990, ApJ, 356, 609

[19] Warner, B.: 1987, MNRAS, 227, 23 